\title{
32 Plant Growth-Promoting Bacteria as an Alternative Strategy for the Amelioration of Salt- Stress Effects in Plants
}

\author{
Živko Jovanović and Svetlana Radović
}

\section{CONTENTS}

32.1 Plant Growth-Promoting Bacteria and Salt

Tolerance in Plants

32.1.1 Mechanisms of PGPB-Mediated Salt-Stress Tolerance...............484

32.1.1.1 Synthesis of Plant Growth Regulators .......................4 484

32.1.1.2 Volatile Organic Compounds ....................................487

32.1.1.3 Exopolysaccharides ............................................... 488

32.1.1.4 Osmoprotectants.....................................................48

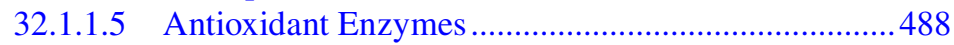

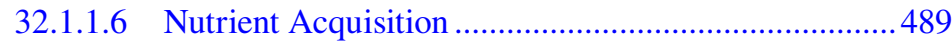

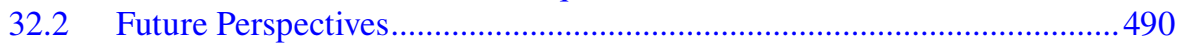

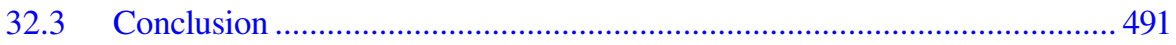

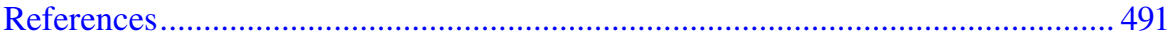

\subsection{PLANT GROWTH-PROMOTING BACTERIA AND SALT TOLERANCE IN PLANTS}

Plant growth-promoting bacteria (PGPB) represent a group of rhizospheric and/ or endophytic bacteria that colonize interior or exterior of roots. Although most of these belong to the genera and Bacillus and Pseudomonas, they may also belong to the Microbacterium, Pantoea, Rhizobium, Burkholderia, Methylobacterium, Azospirillum, Poenibacillus, Micorcoccus and Variovorax. All of them could improve tolerance to host plants during abiotic stresses (Akram et al. 2016; Shahid et al. 2018; Abbas et al. 2019). Those bacteria could improve plant growth under normal conditions, as well as enhance tolerance against salinity stress by various mechanisms. Those mechanisms could be divided into two groups - direct and indirect mechanisms. Direct mechanisms represent improving the bioavailability of different mineral nutrients, such as iron (for siderophore production) and phosphorus 
(phosphate solubilization), nitrogen fixation, production of different phytohormones (indole-acetic acids, auxins, ethylen). Among the indirect mechanisms are the activation of antioxidative defense, production of exopolysaccharides (EPS) and compatible osmolytes and the biosynthesis of volatile organic compounds (VOC) (Yang et al. 2009; Numan et al. 2018; Abbas et al. 2019). PGPB are able to activate "memory" defence in plants and induce response against salinity through microbe-associated molecular patterns. Such mechanisms, providing tolerance in stress conditions, are known as "induced systemic tolerance - IST" (Abbas et al. 2019). Interestingly, PGPB able to activate IST could be salt-tolerant, also, but this is not obligatory.

\subsubsection{Mechanisms of PGPB-Mediated Salt-Stress Tolerance}

PGPB are able to use a plethora of mechanisms, which directly or indirectly ameliorate salt-stress effects in plants (Hashem et al. 2016; Egamberdieva et al. 2019). All these mechanisms act synergistically in order to cope with emerging stress, activating different protection mechanisms, such as antioxidative systems and osmoprotectants.

\subsubsection{Synthesis of Plant Growth Regulators}

Many PGPB have the ability to release phytohormones, such as gibberelic acids (GA), auxins (indole-3-acetic acid - IAA, dominantly), cytokinins (CK) and abscisic acid (ABA). Apart from these hormones, some PGPB strains could modulate plant hormone status by releasing metabolites as well as the enzymes, such as 1-aminocyclopropane-1-carboxylase (ACC) deaminase. Together these can play essential roles in ameliorating salt-stress effects in plants. Acting as specific regulators, these molecules are able to modulate plant metabolism and morphology.

\subsection{Auxins}

Many strains of PGPB can produce auxins, such as indole-3-butyric acid or indole3-acetic acid (IAA), or their precursors (Martinez-Morales et al. 2003; Spaepen et al. 2007; Numan et al. 2018). IAA is the most important and the most investigated auxin, acting as a key player in many processes, like cell division, differentiation and extension, but also in gravitropic and phototropic responses (Korasick et al. 2013) and circadian rhythms (Vinterhalter et al. 2015). This hormone can be produced in the tryptophan-based pathway via the formation of indole-3-pyruvic acid or indole-3-acetamide (Vaishnav et al. 2016). It is interesting that plant root cells secrete tryptophan, produced in the phenylpropanoid pathway from chorismate, and secreted amino acid is taken up by microorganisms situated in the rhizosphere. Production of IAA by PGPB is one of the most common and widely studied bacterial signaling molecules involved in plant-microbe interaction. There is a lot of experimental data confirming the importance of IAA-producing PGPB in minimizing the deleterious effects of salinity on crop plants. Generally, plants inoculated with IAA-producing PGPB grow more main roots and laterals, due to the effects of the hormone on apical meristems. These changes increase plant access to soil nutrients (Manulis et al. 1994; Nicolas et al. 2004). In addition, inoculated plants have more leaves, as IAA is also the main auxin promoting shoot 
growth (Malhotra and Srivastava 2009). All these effects of IAA-producing PRGP on plants are considered as adaptive responses to salinity (Vaishnav et al. 2016). Experiments on Phaseolus vulgaris exposed to $50 \mathrm{mM} \mathrm{NaCl}$ showed that application of Azospirillum brasiliense could alleviate the negative effects of stress, by encouraging higher branching of roots and activating the production of flavonoids (Dardanelli et al. 2008). Yao et al. (2010) showed that Pseudomonas putida was able to modulate production of IAA in plant tissues, increasing growth parameters in cotton plants. Some PGPB, like salt-tolerant Streptomyces isolates that produce IAA, were able to improve the root system of wheat plants exposed to salt stress (Sadeghi et al. 2012). Bacillus amyloliqefaciens SQR 9 was able to enhance saltstress tolerance in maize seedlings exposed to $100 \mathrm{mM} \mathrm{NaCl}$. Maize seedlings inoculated with that bacteria strain showed not only increased total chlorophyll and soluble sugar contents, but also enhanced glutathione content. In addition, SQR9 inoculation activates peroxidase (POD) and catalase (CAT) and reduced the ABA level induced by salinity. As a consequence of plant-microbe interaction, upregulation of many of the genes (RBCS, RBCL, NCED, HKT1, NHX1, NHX2 and NHX3) involved in photosynthesis and metabolism has been observed (Chen et al. 2016; Ilangumaran and Smith 2017). In Arabidopsis and tomato (Solanum lycopersicum) plants, inoculation with Enterobacter sp. EJ01 (isolated from a halophyte) improved the tolerance of plants to salt stress (200 $\mathrm{mM} \mathrm{NaCl})$. In these plants, inoculation with EJ01 activated the expression of genes involved in dehydration, such as DRE-binding proteins DREB2b, LEA genes RAB18 and a gene involved in biosynthesis of proline - P5CS1 (Ilangumaran and Smith 2017). More recent experiments have shown that the salt-tolerant PGPB Leclercia adecarboxylata regulates sugar biosynthesis, the production of different organic acids and chlorophyll fluorescence in tomato under salt stress (Kang et al. 2019). Those effects are also accomplished via IAA.

\subsection{Abscisic Acid}

Although it has been proposed that many PGPB produce ABA in vitro, there are relatively few studies about the role of $\mathrm{ABA}$ in plant-microbe interactions. In addition, it is not clear whether ABA, produced by bacteria, can modulate ABA status in plants exposed to enhanced salinity (Dodd et al. 2010; Ilangumaran and Smith 2017). Some reports have shown that PGPB inhibit ABA production in salt-stressed plants, while others claim that bacteria enhance the accumulation of ABA, providing better conditions for plant survival under salt stress (Vaishnav et al. 2016). However, the experimental data show that PGPB modulate not only ABA biosynthesis, but also ABA-mediated signaling pathways. In wheat, inoculation with Dietzia natronolimnaea STR1 induced salinity tolerance in plants exposed to $150 \mathrm{mM} \mathrm{NaCl}$, via modulation of an ABA-signaling cascade. Inoculation upregulates TaABARE (an ABA-responsive gene) and TaOPRI (the 12-oxophytodienoate reductase gene), which lead to the activation of TaMYB and TaWRKY, and finally upregulated the expression of the stress-related gene TaST (salt stress-induced). Inoculation of cucumber (Cucumis sativus) with Burkholderia cepacia SE4, Promicromonospora sp. SE188 and Acinetobacter calcoaceticus SE370 provided better performance (higher biomass) of plants grown at $120 \mathrm{mM} \mathrm{NaCl}$. It was interesting that inoculated 
plants had a lower ABA level compared with control (non-inoculated) plants, as well as an increased water potential and decreased electrolyte leakage. In addition, inoculation increased salycilic acid and giberellin content (Kang et al. 2014). Inoculation of cotton (Gossypium hirsutum) seeds with Pseudomonas putida Rs-198 increased plant biomass and reduced ABA level. Non-inoculated plants showed a higher accumulation of ABA in leaves (Yao et al., 2010). Barnawal et al. (2017) observed that, in wheat plants, inoculation with B. subtilis LDR2 and Arthrobacter protophormiae SA3 increased ABA content under salt-stress conditions $(100 \mathrm{mM} \mathrm{NaCl})$. That effect was accomplished by upregulation of the TaCTRI (Serine/Threonine protein kinase - ethylene responsive) and TaDRE2 (drought-responsive element) genes.

\subsection{Gibberellin}

There are a few data about the gibberellin-producing PGPB, especially about their role in acquiring salt-stress tolerance in plants. It is well known that some plants, inoculated with different Rhizobium strains (GA7 producers), have longer roots (Numan et al. 2018). Cassán et al. (2014) showed that inoculation of wheat and soybean with GA-producing A. brasilense enhanced plant growth.

\subsection{Cytokinins}

CK are a group of purine-derivates acting as plant hormones: they regulate many processes, like cell division, differentiation of root callus, shoot formation, chloroplast maturation and stomatal conductance in higher plants (Cassán et al. 2014). It is well known that plants maintain their totipotent stem cells in root and shoot meristems due to the action of CK (Howell et al. 2003; Leibfried et al. 2005). Although many experimental data have shown that increased growth of inoculated plants is correlated with cytokinin-producing PGPB (Nieto and Frankenberger 1991; Arkhipova et al. 2005), the role of bacterial CK in salt-stress tolerance is unknown. Egamberdieva (2019) showed that some Pseudomans strains ( $P$. aurantiaca TSAU22, P. extremorientalis TSAU6 and $P$. extremorientalis SAU20) could increase plant growth as well as break salinity $(100 \mathrm{mM} \mathrm{NaCl})$ induced seed dormancy.

\subsection{Ethylene}

Ethylene, the only gaseous plant hormone, is known as a stress hormone. As stress increases the ethylene content in plants, the transcription of auxin response factors is inhibited leading to a perturbation in plant growth. Plants use the Yang cycle for ethylene biosynthesis, in which ACC, as the precursor, is converted into ethylene by the ACC oxidase enzyme (Yoon and Kieber 2013). Salinity stress induces the accumulation of ethylene, as well as its precursor ACC, in the leaves, which results in decreased photosynthesis and foliar senescence (Ghanem et al. 2008). Many PGPB are able to secrete ACC deaminase, an enzyme that restricts ethylene biosynthesis in plants. ACC deaminase converts ACC to ammonia and $\alpha$-ketobutyrate and thereby decreases ethylene levels in plants. Many experimental data show that ACC deaminase-producing PGPB could provide tolerance to plants in saline soil. Thus, cucumber plants are able to grow in saline soil due to the stimulation effects of Stenotrophomonas rhizophila (Egamberdieva et al. 2011). Nadeem et al. (2009) reported that Pseudomonas fluorescens and Enterobacter spp. could improve the 
yield of maize grown in a salt-affected soil. In addition, tomato seedlings, inoculated with Pseudomonas putida UW4, were able to grow in saline conditions $(90 \mathrm{mM}$ $\mathrm{NaCl}$ ). Interestingly, these plants showed increased shoot growth after 6 weeks in saline conditions. In that case, the bacteria used for inoculation, although producing ACC deaminase, were able to up regulate the expression of TocGTPase gene. That gene coding is part of the chloroplast protein import apparatus, facilitating the import of proteins involved in stress response (Yan et al. 2014). Peanut tolerance to saline condition has been improved by inoculation with Brachybacterium saurashtrense (JG-06), Brevibacterium casei (JG-08) and Haererohalobacter (JG-11), ACCproducing PGPB (Shukla et al. 2012). Apart from its role in decreasing ethylene level in plants, preventing them from senescence, it has been found that ACC deaminase producing PGPB strains could have other effects on plants, such as the production of pigments under drought and salt stress, the biosynthesis of compatible solutes and the stabilization of membranes (Tiwari et al. 2018). In pea plants (Pisum sativum cv. Alderman), inoculated with Variovorax paradoxus $5 \mathrm{C}-2$, under salt-stress conditions -70 and $130 \mathrm{mM} \mathrm{NaCl}$, there was an increased $\mathrm{K}^{+} / \mathrm{Na}^{+}$ratio in shoots. This is very important for pea survival under saline conditions, as experimental data have shown that pea varieties have different sensitivity to salinity (Miljuš-Djukić et al. 2013). These strains could also have the effects on nodule formation in legume crops (Ahmad et al. 2011; Egamberdieva et al. 2019). PGPB that produce both ACC deaminase and auxin (IAA) have a great potential for plant protection from different stresses. Accumulation of IAA activates the enzyme ACC synthase, increasing the ACC level and subsequently ethylene level. The excess ACC could be destroyed by ACC-deaminase producing PGPB, allowing the promotion of plant growth under stress conditions mediated by IAA (Glick 2012; Ilangumaran and Smith 2017).

\subsubsection{Volatile Organic Compounds}

VOC play one of the crucial roles in plant-microorganism interactions. Due to high vapor pressure, these compounds can enter the atmosphere as vapors. VOCproducing PGPB are able to modulate many signaling pathways in plants, resulting in the promotion of growth and the activation of induced systemic resistance - ISR (Ryu et al. 2004; Zhang et al. 2008; Numan et al. 2018). Using a microarray, Zhang et al. (2007) showed that the VOC of B. subtilis GB03 in A. thaliana seedlings altered the expression of more than 600 genes. Under saline conditions, VOCs were able to upregulate the expression of HKT1 - the high affinity $\mathrm{K}^{+}$transporter, thus lowering accumulation of $\mathrm{Na}^{+}$in plant tissues (Zhang et al. 2008). Pseudomonas simiae AU, a VOC producing PGPB, was able to induce salt tolerance in soybean (Glycine max) exposed to high salinity $(100 \mathrm{mM} \mathrm{NaCl})$ by decreasing the accumulation of sodium cations in roots and increasing the accumulation of proline. Analysis of proteins revealed upregulation of different vegetative storage proteins involved in $\mathrm{Na}^{+}$homeostasis and the RuBisCO large subunit (Vaishnav et al. 2016). Ledger et al. (2016) showed that VOCs produced by Paraburkholderia phytofirmanas PsJN induced salinity tolerance in vitro and in the soil. Also, all growth parameters, such as fresh weight, length of primary roots and rosette area were higher in Arabidopsis plants treated with VOCs comparing with non-inoculated plants (Ledger et al. 2016). 


\subsubsection{Exopolysaccharides}

EPS or surface polysaccharides secreted by bacteria are responsible for bacterial attachment to root surfaces, soil particles as well as to other bacteria. Some of PGPB secrete EPS (Tewari and Arora 2014; Khan and Bano 2019). It is important that EPS could act as a barrier around plant roots, thus supporting plant growth under saline conditions (Vaishnav et al. 2016). Inoculation of chickpea (Cicer arietinum var. CM-98) with EPS-producing PGPB Halomonas variabilis HT1 and Planococcus rifietoensis $\mathrm{RT} 4$ resulted in increased plant growth at $200 \mathrm{mM} \mathrm{NaCl}$ (Qurashi and Sabri 2012). Yang et al. (2016) inoculated quinoa (Chenopodium quinoa) seeds with Enterobacter sp. MN17 and Bacillus sp. MN54 and showed plant survival under saline irrigation conditions $(400 \mathrm{mM} \mathrm{NaCl})$. Recently, it has been shown that salttolerant EPS producing $B$. subtilis subsp. inaquosorum and Marinobacter lypoliticus SM19 reduced drought and salinity stress effects in wheat (Atouei et al. 2019). In A. thaliana, EPS producing Pseudomonas sp. was able to upregulate the LOX2 gene which encodes a lipoxygenase. This enzyme is a component of the jasmonic acid biosynthesis pathway (Chu et al. 2019).

Apart from exopolysaccharides, many PGPB produce lipo-chitooligosaccharides. These molecules are secreted by rhizobia as Nod-factors (NFs) and their secretion is induced by flavonoids present in root exudates. NFs, thus, initiate nodule formation (Ilangumaran and Smith 2017). Miransari and Smith (2009) reported that inoculation of soybean plants with Bradyrhizobium japonicum $532 \mathrm{C}$ enhanced nodulation and growth under mild salinity stress $(36 \mathrm{mM}$ and $61 \mathrm{mM} \mathrm{NaCl})$.

\subsubsection{Osmoprotectants}

Salt stress is basically a two-component stress and plant growth and development are first affected by the osmotic impacts of salt stress. The accumulation of salt ions leads to a decrease in the osmotic balance in plants. Plants accumulate compatible osmolytes to improve plant-water relations and maintain cell structures protected from osmotic shock. Many PGPB also use this mechanism for protection against osmotic stress (Ilangumaran and Smith 2017). Choudhary et al. (2012) reported that many PGPB, such as Burkholderia, Arthobacter, Bacillus and Pseudomonas, are able to enhance proline levels in plants under abiotic stress. Some endophytic bacteria, producing ACC deaminase and IAA, are able to increase proline content in sweet pepper (Capsicum annum). Maize plants (Zea mays) inoculated with Rhizobium and Pseudomonas accumulated more proline comparing with control (non-inoculated) plants (Bano and Fatima 2009). According to the experimental data, it is evident that the accumulation of proline under abiotic stress is a result of the activation of the pyrroline-5-carboxylate synthase (P5CS) gene; thus, bacterial treatment activates the expression of P5CS gene (Kumari et al. 2015). Some PGPB also produce polyamines, such as spermidine (Bacillus megaterium BOFC15) and soluble sugars (trehalose). Many of PGPB have genes for the biosynthesis of trehalose (Qin et al. 2018; Shim et al. 2019).

\subsubsection{Antioxidant Enzymes}

Many reports have shown that inoculation of plants with PGPB reduced the oxidative stress in plants and its deleterious effects (Manaf and Zayed 2015; Islam et al. 2016). 
PGPB strains are able to activate and even increase the concentration of antioxidative enzymes. Under saline conditions, the activity of soluble POD, superoxide dismutase, CAT and glutathione reductase has been increased by PGPB (Jha and Subramanian 2013; Sen and Chandrasekhar 2015; Ansari et al. 2019). Plant inoculation with Azospirillum lipoferum FKI induced an increasing level of antioxidant gene transcripts (El-Esawi et al. 2019). Kohler et al. (2009) reported that lettuce plants inoculated with PGPB showed increased activity of CAT under salinity stress. In Jatropha plants exposed to salt stress, Patel and Saraf (2013) observed an increased level of APX and CAT activity in leaves. Although many reports have claimed that plants inoculated with PGPB showed increased activities of antioxidative enzymes, the exact mechanism for enzyme activation remains unknown.

\subsubsection{Nutrient Acquisition}

Salinity can affect plant growth and productivity due to a limitation of nutrients in the soil. As salinity changes the $\mathrm{pH}$ of soil, some nutrients become less available for plants. PGPB are able to solubilize different nutrients making them available for plants. PGPB exert these effects by different mechanisms, such as siderophore production, increased phosphorous solubilization, organic and inorganic phosphate solubilization and non-symbiotic nitrogen fixation.

Siderophores are agents able to chelate iron and represent an important trait for the promotion of plant growth. Siderophores produced by different PGPB have high affinity for iron, thus preventing the proliferation of phytopathogens (Numan et al. 2018). Siderophores are secreted in the rhizosphere, so plant roots can uptake iron from them via two mechanisms - by chelate degradation or by direct uptake (Rajkumar et al. 2010). In saline soil, the availability of $\mathrm{Fe}^{3+}$ ions can be reduced due to changes in $\mathrm{pH}$ (Thomine and Lanquar 2011); plant inoculation with siderophore-producing PGPB can, therefore, help plants to overcome the nutrient stress caused by high salinity.

Phosphorus is a crucial macronutrient for plants. In organic form, it is present as inositol phosphates, phosphoesters, phosphodiesters and phosphotriesters (Sindhu et al. 2010). A large part of the soil phosphoros pool is immobilized and unavailable for plants (Numan et al. 2018). PGPB can play an important role in the transformation of phosphoros, making it available for plants. Phosphate solubilizing bacteria belong to the genera Bacillus, Pseudomonas, Brevibacterium, Serratia, Xanthomonas, Corynebacterium and Alcaligenes (Sindhu et al. 2010). These bacteria are able to hydrolize unavailable forms of phosphorus into more available forms. As salinity can cause the precipitation of available phosphorus, PGPB could solubilize precipitated forms, providing nutrients to plants under salinity stress.

PGPB have the ability to provide other nutrients to plants, such as nitrogen, potassium, sulfur and zinc (Vaishnav et al. 2016). Some PGPB can enhance the nodulation process, especially in saline soil, which is important because Rhizobium are often not effective for the induction of nodulation under saline conditions (Ahmad et al. 2011). 


\subsection{FUTURE PERSPECTIVES}

In order to successfully use PGPB strains in the region affected by increased salinity the best way is to use halotolerant bacteria for plant and/or seed inoculation. These PGPB should be isolated from salt-affected soils, which make them able to colonize roots under salinity (Paul and Nair 2008; Vaishnav et al. 2016). Ramadoss et al. (2013) demonstrated that five PGPB halotolerant bacteria could ameliorate salt stress in wheat plants. In these experiments, they used $320 \mathrm{mM} \mathrm{NaCl}$ for inducing salt stress and observed that inoculation was able to increase root length by up to $71.7 \%$. Apart from the use of halotolerant bacteria, it is speculated that is possible to use PGPB which are not halotolerant (tested in laboratory conditions with growing medium supplemented with $\mathrm{NaCl}$ ) for the enhancement of plant growth under saline conditions. In that case it should be the use of bacterial cultures for seed inoculation, so called "seed priming". In this way, PGPB could activate different mechanisms in plants, including ISR, making them more tolerant to salinity. Some plant seeds, inoculated with non-halotolerant PGPB, were able to germinate faster under salt stress, compared with non-inoculated control seeds.

The commercialization of PGPB strains is a complex process, which includes many stages - from isolation, screening and testing in laboratory conditions, up to testing in the field, estimating their efficacy and making adequate formulations. It is necessary also to test the viability of formula and industrial production, etc. (Bhattacharyya and Jha 2012; Vaishnav et al. 2016).

Many of the mechanisms of osmo-adaptation of PGPB still need to be elucidated; establishing these could have a big impact on the process of improvement of productivity in agriculturally important plants grown in saline agro-ecosystem (Paul 2013). Further work is also required to investigate the regulatory networks which provide salt (and other) stress tolerance to bacteria. Also, plant-microbe interaction, particularly under saline condition, should be deeper investigated. Although many mechanisms are well documented, the possible interaction among them is still controversial. A deeper analysis of the bacterial communities associated with plant roots, as well as examination of the dynamic changes of microbiomes under saline condition could provide valuable data.

It is very important that the research findings obtained under laboratory conditions be extended to field trials and in different geographical regions. Also important is that this research should be conducted on different plants, even on different cultivars, which is a major challenge. The major issue for the end-users is using developed bioinoculant formula on many different plants. Having in mind that PGPB are diverse, in respect to their plant-growth promoting effects, the recommendation is to use no single PGPB strains, but several strains or consortia. Using diverse microbes in consortia formulations is potentially a promising strategy for the alleviation not only of salinity (and other abiotic) stress in plants, but also biotic stress - phytopathogens (Egamberdieva et al. 2019).

PGPB used for enhancing the plant productivity in saline agro-ecosystem have the potential to help in achieving food security and improving global food production. Moreover, they can change the quality of soil and help in combating the adverse effects of climate change (Arora 2019). 


\subsection{CONCLUSION}

Salt stress is still a major problem for agriculture, limiting the yield of many agriculturally important crops. Although plant-growth promoting bacteria, including halotolerant types, represent a promising tool for the alleviation of salt-stress effects on plants, a lot is yet to be explored, at the biochemical as well as at the molecular levels. Apart from helping plants, PGPB in saline agro-ecosystem have the potential to improve soil qualities, like soil fertility. These microorganisms, used as biocontrol agents, as a part of alternative green biotechnologies, should be extensively used in the future not only in saline soils, but also in other marginal soil types. Many issues, including problems with the formulation and production of final products, as well as potential legal and social impacts, should be further analyzed.

\section{REFERENCES}

Abbas R, Rasul, Aslam K, Baber M, Shahid M, Mubeen F, Naqqash, T. 2019. Halotolerant PGPR: A hope for cultivation of saline soils. Journal of King Saud University Science 31: 1195-1201.

Ahmad M, Zahir ZA, Asghar HN, Asghar M. 2011. Inducing salt tolerance in mung bean through coinoculation with rhizobia and plant-growth-promoting rhizobacteria containing 1-aminocyclopropane-1-carboxylate deaminase. Canadian Journal of Microbiology 57: 578-589.

Akram MS, Shahid M, Tariq M, Azeem M, Javed MT, Saleem S, Riaz S. 2016. Deciphering Staphylococcus sciuri SAT-17 Mediated Anti-oxidative Defense Mechanisms and Growth Modulations in Salt Stressed Maize (Zea mays L.). Frontiers in Microbiology 7:867.

Ansari FA, Ahmad I, Pichtel J. 2019. Growth stimulation and alleviation of salinity stress to wheat by the biofilm forming Bacillus pumilus strain FAB10. Applied Soil Ecology 3: 45-54.

Arkhipova TN, Veselov SU, Melentiev AI, Martynenko EV, Kudoyarova GR. 2005. Ability of bacterium Bacillus subtilis to produce cytokinins and to influence the growth and endogenous hormone content of lettuce plants. Plant Soil 272: 201-209.

Arora NK. 2019. Impact of climate change on agriculture production and its sustainable solutions. Environmental Sustainability 2:95-96.

Atouei MT, Pourbabaee AA, Shorafa M. 2019. Alleviation of salinity stress on some growth parameters of wheat by exopolysaccharide-producing bacteria. Iranian Journal of Science and Technology Transaction A 43: 2725-2733.

Bano A, Fatima M. 2009. Salt tolerance in Zea mays (L.) following inoculation with Rhizobium and Pseudomonas. Biology and Fertility of Soils 45: 405-413.

Barnawal D, Bharti N, Maji D, Chanotiya CS, Kalra A. 2014. ACC deaminase-containing Arthrobacter protophormiae induces $\mathrm{NaCl}$ stress tolerance through reduced ACC oxidase activity and ethylene production resulting in improved nodulation and mycorrhization in Pisum sativum. Journal of Plant Physiology 171:884-894.

Bhattacharyya PN, Jha DK. 2012. Plant growth-promoting rhizobacteria (PGPR): Emergence in agriculture. World Journal of Microbiololgy and Biotechnology 28: 1327-1350.

Cassán F, Vanderleyden J, Spaepen S. 2014. Physiological and agronomical aspects of phytohormone production by model plant-growth-promoting rhizobacteria (PGPR) belonging to the genus Azospirillum. Journal of Plant Growth Regulation 33:440-459. 
Chen L, Liu Y, Wu G, Veronican Njeri K, Shen Q, Zhang N. et al. 2016. Induced maize salt tolerance by rhizosphere inoculation of Bacillus amyloliquefaciens SQR9. Physiologia Plantarum 158: 34-44.

Choudhary D. 2012. Microbial rescue to plant under habitat-imposed abiotic and biotic stresses. Applied Microbiology and Biotechnology 96:1137-1155.

Chu TN, Tran BTH, Van Bui L, Hoang MTT. 2019. Plant growth-promoting rhizobacterium Pseudomonas PS01 induces salt tolerance in Arabidopsis thaliana. BMC Research Notes 12:11.

Dardanelli MS, Fernández de Córdoba FJ, Espuny MR, Rodríguez-Carvajal MA, Soría-Díaz ME, Gil-Serrano AM, Okon Y, Megías M. 2008. Effect of Azospirillum brasilense coinoculated with Rhizobium on Phaseolus vulgaris flavonoids and Nod factor production under salt stress. Soil Biology and Biochemistry 40:2713-2721.

Dodd IC, Zinovkina NY, Safronova V I, Belimov AA. 2010. Rhizobacterial mediation of plant hormone status. Annals of Applied Biology 157: 361-379.

Egamberdieva D, Wirth S, Bellingrath-Kimura SD, Mishra J, Arora NK. 2019. Salt- tolerant plant growth promoting rhizobacteria for enhancing crop productivity of saline soils. Frontiers in Microbiology 10:2791.

Egamberdieva, D, Kucharova, Z, Davranov, K, Berg, G, Makarova, N, Azarova T, Chebotar V, Tikhonovich I, Kamilova F, Validov S, Lugtenberg B. 2011. Bacteria able to control foot and root rot and to promote growth of cucumber in salinated soils. Biology and Fertility of Soils 47: 197-205.

El-Esawi MA, Alaraidh IA, Alsahli AA, Alamri SA, Ali HM, Alayafi AA. 2018. Bacillus firmus (SW5) augments salt tolerance in soybean (Glycine max L.) by modulating root system architecture, antioxidant defense systems and stress-responsive genes expression. Plant Physiology and Biochemistry 132: 375-384.

Ghanem ME, Albacete A, Martínez-Andújar C, Acosta M, Romero-Aranda R, Dodd IC, Lutts S, Pérez-Alfocea F. 2008. Hormonal changes during salinity-induced leaf senescence in tomato (Solanum lycopersicum L.) Journal of Experimental Botany 59:3039-3050.

Glick B. 2012. Plant growth-promoting bacteria: Mechanisms and applications. Scientifica. 2012:1-15.

Hashem A, Abd_Allah EF, Alqarawi AA, Al-Huqail AA, Wirth S, Egamberdieva D. 2016. The interaction between arbuscular mycorrhizal fungi and endophytic bacteria enhances plant growth of Acacia gerrardii under salt stress. Frontiers in Microbiology 7:1089.

Howell SH, Lall S, Che P. 2003. Cytokinins and shoot development. Trends in Plant Science 8: 453-459.

Ilangumaran G, Smith DL. 2017. Plant growth promoting rhizobacteria in amelioration of Salinity stress: a systems biology perspective. Frontiers in Plant Science 8:1768.

Islam F, Yasmeen T, Arif MS, Ali S, Ali B, Hameed S et al. 2016. Plant growth promoting bacteria confer salt tolerance in Vigna radiata by up-regulating antioxidant defense and biological soil fertility. Plant Growth Regulation 80: 23-36.

Jha Y, Subramanian RB. 2013. Paddy plants inoculated with PGPR show better growth physiology and nutrient content under saline condition. Chilean Journal of Agricultural Research 73: 213-219.

Kang SM, Radhakrishnan R, Khan AL, Kim MJ, Park JM, Kim BR, Shin DH, Lee IJ.2014. Gibberellin secreting rhizobacterium, Pseudomonas putida $\mathrm{H}$ - 2- 3 modulates the hormonal and stress physiology of soybean to improve the plant growth under saline and drought conditions. Plant Physiology and Biochemistry 84: 115-124.

Kang SM, Shahzad R, Bilal S, Khan A L, Park YG, Lee K E et al. 2019. Indole-3-acetic-acid and ACC deaminase producing Leclercia adecarboxylata MO1 improves Solanum lycopersicum L. growth and salinity stress tolerance by endogenous secondary metabolites regulation. BMC Microbiology 19:80. 
Khan N, Bano A. 2019. Exopolysaccharide producing rhizobacteria and their impact on growth and drought tolerance of wheat grown under rainfed conditions. PLoS One 14: $\mathrm{e} 0222302$.

Kohler J, Hernandez JA, Caravaca F, Roldan A. 2009. Induction of antioxidant enzymes is involved in the greater effectiveness of a PGPR versus AM fungi with respect to increasing the tolerance of lettuce to severe salt stress. Environmental and Experimental Botany 65: 245-252.

Korasick DA, Enders TA, Strader LC. 2013. Auxin biosynthesis and storage forms. Journal of Experimental Botany 64: 2541-2555.

Kumari S, Vaishnav A, Jain V, Choudhary DK. 2015. Bacterial-mediated induction of systemic tolerance to salinity with expression of stress alleviating enzymes in soybean (Glycine max L. Merrill). Journal of Plant Growth Regulation 34: $558-573$.

Ledger T, Rojas S, Timmermann T, et al. 2016. Volatile-mediated effects predominate in Paraburkholderia phytofirmans growth promotion and salt stress tolerance of Arabidopsis thaliana. Frontiers in Microbiology 7:1838.

Leibfried A, To JPC, Busch W, Stehling S, Kehle A, Demar M, Kieber JJ, Lohmann JU. 2005. WUSCHEL controls meristem function by direct regulation of cytokinin-inducible response regulators. Nature 438: 1172-1175.

Malhotra M, Srivastava S. 2009. Stress-responsive indole-3-acetic acid biosynthesis by Azospirillum brasilense SM and its ability to modulate plant growth. European Journal of Soil Biology 4573-4580.

Manaf HH, Zayed MS. 2015. Productivity of cowpea as affected by salt stress in presence of endomycorrhizae and Pseudomonas fluorescens. Annals of Agricultural Sciences 60: 219-226.

Manulis S, Shafri H, Epstein E, Lichter A, Barash I. 1994. Biosynthesis of Indole 3-acetic acid via the indole 3-acetamide pathway in Streptomyces spp. Microbiology 140: $1045-1050$.

Martínez-Morales LJ, Soto-Urzúa L, Baca BE, Sánchez-Ahédo, JA. 2003. Indole-3-butyric acid (IBA) production in culture medium by wild strain Azospirillum brasilense. FEMS Microbiology Letters, 228: 167-173.

Miljuš-Djukić J, Stanisavljević N, Radovic S, Mikic A, Maksimovic V, Jovanović Ž. 2013. Differential response of three contrasting pea (Pisum arvense, P. sativum and P. fulvum) species to salt stress: assessment of variation in antioxidative defence and miRNA expression. Australian Journal of Crop Science 7:2145-2153.

Miransari M, Smith D. 2009 Alleviating salt stress on soybean (Glycine max (L.) Merr.)Bradyrhizobium japonicum symbiosis, using signal molecule genistein. European Journal of Soil Biology 45:146-152.

Nadeem SM, Zahir ZA, Naved M, Arshad M. 2009. Rhizobacteria containing ACCdeaminase confer salt tolerance in maize grown on salt-affected fields. Canadian Journal of Microbiology 55: 1302-1309.

Nicolás L, Ines J, Acosta M, Sánchez-Bravo J. 2004. Role of basipetal auxin transport and lateral auxin movement in rooting and growth of etiolated lupin hypocotyls. Physiologia plantarum 121: 294-304.

Nieto K, Frankenberger W.1991. Influence of adenine, isopentyl alcohol and Azotobacter chroococcum on the vegetative growth of Zea mays. Plant and Soil 135: 213-221.

Numan M, Bashir S, Khan Y, Mumtaz R, Shinwari ZK, Khan AL, Khan A, AL-Harrasi A. 2018. Plant growth promoting bacteria as an alternative strategy for salt tolerance in plants: A review. Microbiology Research 209: 21-32.

Patel T, Saraf M. 2017. Biosynthesis of phytohormones from novel rhizobacterial isolates and their in vitro plant growth-promoting efficacy. Journal of Plant Interactions 12: 480-487. 
Paul D. 2013. Osmotic stress adaptations in rhizobacteria. Journal of Basic Microbiology 53: 101-110.

Paul D, Nair S. 2008. Stress adaptations in a plant growth promoting rhizobacterium (PGPR) with increasing salinity in the coastal agricultural soils. Journal of Basic Microbiology 48:378-384.

Qin S, Feng WW, Zhang YJ, Wang TT, Xiong YW, Xing K. 2018. Diversity of bacterial microbiota of coastal halophyte Limonium sinense and amelioration of salinity stress damage by symbiotic plant growth-promoting actinobacterium Glutamicibacter halophytocola KLBMP 5180. Applied and Environmental Microbiology 84: e1533-e1518.

Qurashi AW, Sabri, AN. 2012. Bacterial exopolysaccharide and biofilm formation stimulate chickpea growth and soil aggregation under salt stress. Brazilian Journal of Microbiology 43: 1183-1191.

Rajkumar M, Ae N, Prasad MNV, Freitas H. 2010. Potential of siderophore-producing bacteria for improving heavy metal phytoextraction. Trends in Biotechnology 28; 142-149.

Ramadoss D, Lakkineni VK, Bose P, Ali S, Annapurna K. 2013. Mitigation of salt stress in wheat seedlings by halotolerant bacteria isolated from saline habitats. Springer Plus, 2: $1-7$.

Ryu CM, Farag MA, Hu CH, Reddy MS, Kloepper JW, Paré PW. 2004. Bacterial volatiles induce systemic resistance in Arabidopsis. Plant Physiology 134:1017-1026.

Sadeghi A, Karimi E, Dahaji PA, Javid MG, Dalvand Y, Askari H. 2012. Plant growth promoting activity of an auxin and siderophore producing isolate of Streptomyces under saline soil conditions. World Journal of Microbiology and Biotechnology 28: 1503-1509.

Sen S, Chandrasekhar CN. 2015. Effect of PGPR on enzymatic activities of rice (Oryza sativa L.) under salt stress. Asian Journal of Plant Science and Research 5: 44-48.

Shahid M, Akram MS, Khan MA, Zubair M, Shah SM, Ismail M, Shabir G, Basheer S, Aslam K, Tariq M. 2018. A phytobeneficial strain Planomicrobium sp. MSSA-10 triggered oxidative stress responsive mechanisms and regulated the growth of pea plants under induced saline environment. Journal of Applied Microbiology, 124: 1566-1579.

Shim JS, Seo JS, Kim Y, Koo Do Choi Y et al. 2019. Heterologous expression of bacterial trehalose biosynthetic genes enhances trehalose accumulation in potato plants without adverse growth effects. Plant Biotechnology Reports 13: 409-418.

Shukla PS, Agarwal PK, Jha B. 2012. Improved salinity tolerance of (Arachis hypogaea L.) by the interaction of halotolerant plant-growth-promoting rhizobacteria. Journal of Plant Growth Regulation 31: 195-206.

Sindhu SS, Dua S, Verma MK, Khandelwal A. 2010. Growth Promotion of Legumes by Inoculation of Rhizosphere Bacteria. In Microbes for legume improvement, ed. Khan MS, Musarrat J, Zaidi A. Vienna: Springer.

Spaepen S, Vanderleyden J, Remans R. 2007. Indole-3-acetic acid in microbial and microorganism-plant signalling. FEMS Microbiology Reviews 31: 425-448.

Tewari S, Arora NK. 2014. Multifunctional exopolysaccharides from Pseudomonas aeruginosa PF23 involved in plant growth stimulation, biocontrol and stress amelioration in sunflower under saline conditions. Current Microbiology 69: 484-494.

Thomine S, Lanquar V. 2011. Iron Transport and Signaling in Plants. In Transporters and pumps in plant signaling, signaling and communication in plants $7 M$, ed. Geisler and K. Venema, 99-131, Berlin Heidelberg: Springer-Verlag.

Tiwari G, Duraivadivel P, Sharma S, Hariprasad P. 2018. 1-Aminocyclopropane-1-carboxylic acid deaminase producing beneficial rhizobacteria ameliorate the biomass characters of Panicum maximum Jacq. by mitigating drought and salt stress. Scientific Reports 8:17513.

Vaishnav A, Varma A, Tuteja N, Choudhary DK. 2016. PGPR-mediated amelioration of crops under salt stress. In Plant microbe interaction: an approach to sustainable agriculture, ed.D. Choudhari, A.Varna and N.Tuteja, 205-226, Singapore: Springer. 
Vinterhalter D, Vinterhalter B, Miljuš-Djukić J, Jovanović Ž, Orbović V. 2015. (Erratum) Daily changes in the competence for photo- and gravitropic response by potato plantlets. Journal of Plant Growth Regulation 34:440-450.

Yan J, Campbell JH, Glick BR, Smith MD, Liang Y. 2014. Molecular characterization and expression analysis of chloroplast protein import components in tomato (Solanum lycopersicum). PLoS One 9(4): e95088.

Yang A, Akhtar SS, Iqbal S, Amjad M, Naveed M, Zahir ZA et al. 2016. Enhancing salt tolerance in quinoa by halotolerant bacterial inoculation. Functional Plant Biology 43 : $632-642$.

Yang J, Kloepper JW, Ryu CM. 2009. Rhizosphere bacteria help plants tolerate abiotic stress. Trends Plant Sci. 14: 1-4.

Yao L, Wu Z, Zheng Y, Kaleem I, Li C. 2010. Growth promotion and protection against salt stress by Pseudomonas putida Rs-198 on cotton. European Journal of Soil Biology 46: $49-54$.

Yoon GM, Kieber JJ. 2013. 14-3-3 regulates 1-aminocyclopropane-1-carboxylate synthase protein turnover in Arabidopsis. Plant Cell 25:1016-1028

Zhang H, Kim MS, Krishnamachari V, Payton P, Sun Y, Grimson M, et al. 2007. Rhizobacterial volatile emissions regulate auxin homeostasis and cell expansion in Arabidopsis. Planta 226: 839-851.

Zhang H, Kim MS, Sun, Dowd SE, Shi H, Paré P W. 2008. Soil bacteria confer plant salt tolerance by tissue-specific regulation of the sodium transporter HKT1. Molecular PlantMicrobe Interaction 21: 737-744. 


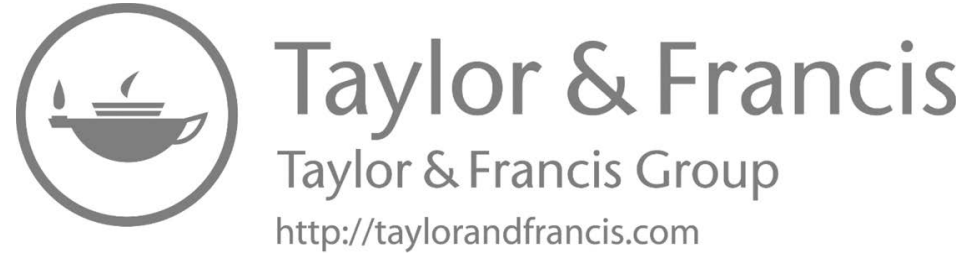

